\title{
Grain Refinement, Stacking Faults and Mechanical Properties of Nanocrystalline Pd and Pd Alloys
}

\author{
C. Kübel*, L. Kurmanaeva*, A. Castrup*, T. Scherer*, J. Ivanisenko* \\ * Institute of Nanotechnology, Karlsruhe Institute of Technology, Hermann-von-Helmholtz-Platz 1, \\ 76344 Eggenstein-Leopoldshafen, Germany
}

Metals with grain sizes in the range of $30 \mathrm{~nm}$ and less exhibit outstanding mechanical properties resulting in new application areas for bulk nanocrystalline (nc) metals. Due to the small grain size, modified or even new deformation mechanism are expected to dominate the mechanical properties, determining hardness, strength and ductility of the material [1]. We are trying to elucidate on the deformation mechanisms in nanocrystalline palladium and palladium alloys by mechanical testing in combination with ex-situ and in-situ TEM analysis, which is further supported by in-situ x-ray diffraction experiments at the synchrotron.

One of the problems for the application of nanocrystalline metals is often the low ductility and the rapid strain localization due to the limited strain hardening capacity. In order to increase the ductility of nc Pd, it was alloyed with Ag to reduce the stacking fault energy and thus increase the dislocation splitting distance, which was expected to lead to an increased dislocation storage and strain hardening [2]. The nanostructural changes associated with the alloying after high pressure torsion (HPT) were characterized by XRD and TEM and showed a reduction of the grain size due to Ag alloying. Furthermore, aberration corrected TEM revealed a high density of partial dislocations (Fig. 1) indicating that the reduced stacking fault energy is at least one of the mechanisms resulting in the improved yield stress and strain hardening rates observed in the nc PdAg alloys (Fig. 2) [3].

\section{References}

[1] J. Ivanisenko, L. Kurmanaeva, J. Weismüller, K. Yang, J. Markmann, H. Rösner, T. Scherer, H.-J. Fecht, Acta Mat. 57 (2008), 3391.

[2] Y.H. Zhao, Y.T. Zhu, X.Z. Liao, Z. Horita, T.G. Langdon, Mater Sci Eng A 463 (2008) 22.

[3] L. Kurmanaevạ, Yu. Ivanisenko, J. Markmann, C. Kübel, A. Chuvilin, S. Doyle, R.Z. Valiev, H.-J. Fecht, Mater Sci Eng A (2010) in press. 




FIG. 1. HRTEM image of stacking faults in HPT processed nc Pd-20\%Ag viewed along $<110>$ direction with corresponding FFT. Several stacking faults are indicated by arrows and two are shown enlarged in A and B. C shows a stacking fault tetrahedron.



FIG. 2. Tensile stress-strain curve of UFG Pd, Pd-10\%Ag, Pd-20\%Ag and Pd-40\%Ag. 